\title{
Effect of Two-component solution-crosslinking Agent on Recycling of waste PU to prepare composites
}

\author{
Xiaohua Gua, Hongxiang Luob, Yan Lic, Fu Li,Rui Cao,Shiwei Lv \\ College of Materials Science and Engineering, Qiqihar University, Qiqihar 161006, China

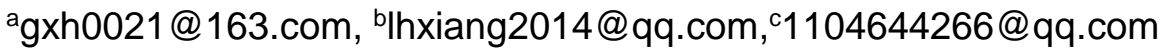

\section{Keywords: waste PU foam, degradation, glass fiber}

Abstract. The waste polyurethane rigid foam was degraded by two-component decrosslinking agent, and a qualified glass fiber reinforced waste polyurethane rigid foam is prepared by compounding the obtained oligomer polyol with a certain percent of glass fibers. The regenerated oligomer polyols were measured for infrared spectroscopy. The foam samples were analyzed for density, water absorption, and compressive strength. The results show that when the mass ratio of DEG/ETA is $1 / 3$, the prepared composite material has the best performance.

\section{Introduction}

Glass fiber (GF) is an inorganic non-metallic material with excellent properties ${ }^{[1]}$, which is applied in various fields of the national economy. The annual consumption of glass fiber is relatively large, and it will produce a large amount of waste and it will be difficult to deal with, and the environment will be more polluted. At the same time, polyurethane materials are developing rapidly in China, increasing at a rate of $20 \%$ to $30 \%$ every year ${ }^{[2]}$. The current annual output has reached more than 20 million tons ${ }^{[3]}$, and its waste is difficult to handle, insoluble and infusible, which brings a lot of problems to enterprises.

Therefore, in this paper,the recycling of glass fiber and polyurethane waste was discussed. In order to explore the best crosslinking agent mass ratios for degradation and optimal production process, the waste polyurethane, as raw material, is decomposed by different mass ratios of two-component crosslinking agents to recycle polyether polyols. The waste polyurethane-glass fiber composites were prepared by using glass fiber, and they are tested and characterized.

\section{Materials and Methods}

\section{Materials}

Waste Polyurethane Rigid Foam; Glass Fiber; Diethylene Glycol, Tianjin Kaitong Chemical Reagent Co. Ltd., Analytical Reagent(AR); Ethanolamine, Tianjin Chemical Reagent No. 1,Analytical Reagent (AR); Polyether (4110), Langfang Quanzheng Chemical Co. Ltd., chemical pure(CP); polyaryl polymethylene isocyanate, Wanhua Chemical Group Co.Ltd., analytical Reagent(AR).

\section{Experimental method}

Using waste polyurethane rigid foams as raw materials, and the waste PU rigid foams were degraded by diethylene glycol (DEG) and ethanolamine (ETA), which are two-component solution-crosslinking agents. The resulting degradation products were subjected to treatment to recycle oligomer polyols and a certain percent of glass fiber were added in it.Then the glass fiber reinforced waste polyurethane rigid foam was prepared. 


\section{Testing and Characterization}

Fourier transform infrared (FT-IR) spectra of samples in $\mathrm{KBr}$ pellets were obtained by a spectrometer (BJO-RAD, FTS-135, USA). The FT-IR spectra were acquired in the wave number range of $400-4000 \mathrm{~cm}^{-1}$.

According to the standard of QB/T 3806-1999, the foam samples were cut into $200 \mathrm{~mm} \times 200$ $\mathrm{mm} \times 20 \mathrm{~mm}$. Thermal conductivity was measured by the thermal conductivity tester instrument (DRPL-III, Shanghai Jiezhun Instrument Co., Ltd.).

According to the standard of GB/T6343-1986, the apparent density was tested after the sample was prepared $72 \mathrm{~h}$ ago.The results for each foam type were averaged from five repeated measurement.

According to the test standard of GB/T8810-88, the size of the sample is $50 \mathrm{~mm} \times 10 \mathrm{~mm} \times 10 \mathrm{~mm}$, and the water absorption rate was measured by distilled water. The mass of the sample before and after soaking were measured, and then the water absorption rate was calculated.

Compressive strength tests were performed on a universal testing machine (WSM-20KN, Changchun Intelligent Instrument Equipment Co., Ltd.) according to the test standard of GB $8813-2008$, and the size of the specimen is $50 \mathrm{~mm} \times 50 \mathrm{~mm} \times 50 \mathrm{~mm}$.

\section{Results and discussion}

Variable-controlling approach was used to study the experiments. When the total amount of solution-crosslinking agent remained unchanged, the mass ratios of DEG/ ETA was in the range of $1 / 3$ to $3 / 1$, and the different degradation experiments were carried out in this condition.The optimal foaming process of the obtained oligomer polyol was studied and the obtained samples were characterized and analyzed.

Fig. 1 shows the results of infrared spectra of the degradation products ( DTG /ETA=1/1, 1/2, and $1 / 3$, mass ratio) and polyether 4110 .

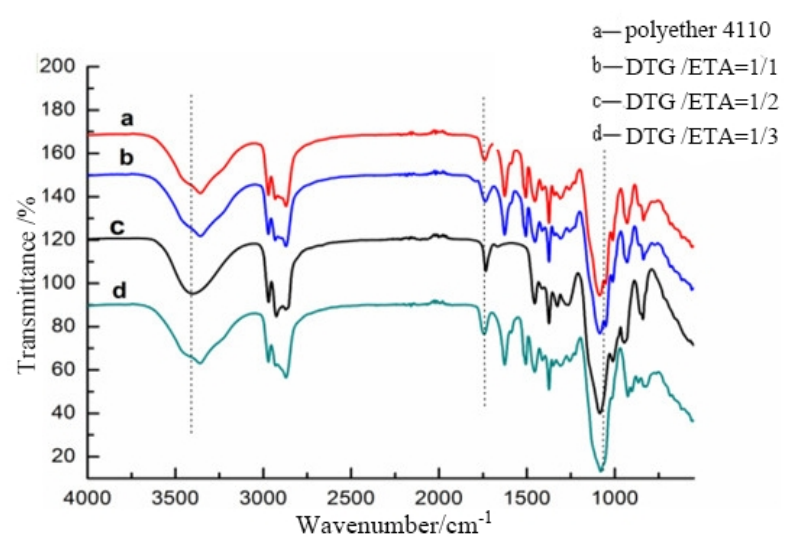

Fig. 1 Infrared spectra of degradation products with different DEG/ETA mass ratio and polyether 4110

Compared with the polyether 4110, the degradation products showed strong absorption bands in the range of $3500-3300 \mathrm{~cm}^{-1}$, which is attributed to alcohol-based hydroxy stretching vibration peaks $^{[4]}$; there is a strong absorption band near $1740 \mathrm{~cm}^{-1}$, which is attributed to a benzene-type overtone peak ${ }^{[5]}$. There is a clear and strong absorption band at $1084 \mathrm{~cm}^{-1}$, which is attributed to a polyether-based polyurethane ether-based absorption peak ${ }^{[6]}$. These data demonstrate that the degradation product is a mixed product of a polyether polyol and an aromatic polyol. From Fig. 1 we can see that the characteristic peak of the foam ( $\mathrm{DEG} / \mathrm{ETA}=1 / 3$, mass radio) is more obvious than others, so when the mass ratio of DEG/ETA is $1 / 3$,it is the best formulation for degrading waste polyurethane rigid foam.

As can be seen from Fig. 2, there are some difference among foam densities. The trend of change is similar, and a large amount of gas is contained in the foam. For the same volume samoles, the higher gas contented, the smaller the density is, and all samples are meet the requirements of national 
standards. The overall conclusion is that when the mass ratio of DEG/ETA is $1 / 3$, the foam is the best,whose cell is the largest and density is the lowest.

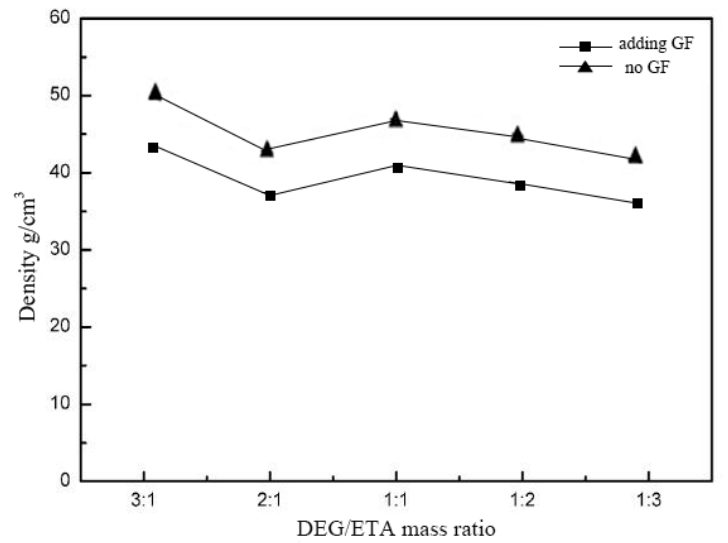

Fig. 2 Density of foam of different type

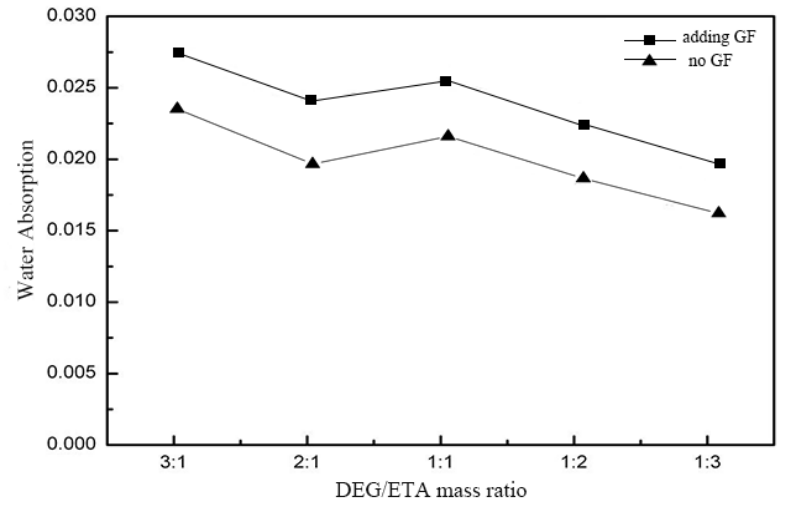

Fig. 3 Water absorption of foam of different type

The water absorption of the foam prepared by the two-component formula was tested and the test results are shown in Fig. 3.

As can be seen from Fig. 3 , the water absorption of the polyurethane rigid foam prepared from the two-component formula meets the national standard. The water absorption of the good foams (DEG/ET $=2 / 1,1 / 2$, and $1 / 3$, mass ratio) show a decreasing trend, indicating that the heat and insulation performance of the three groups are upward trend. The lower the water absorption, the more complete the cell structure of the foam, the higher the closed cell rate of the foam, and the more gas locked up. For foam of lower water absorption,its insulation performance is better.From the density, water absorption, and pressure resistance of the two-component formula foam, we can see that the foam (DEG/ETA=1/3, mass radio) showing superior overall performance.

In order to further analyze the properties of the prepared glass fiber reinforced waste polyurethane rigid foam, the thermal conductivity of the two-component foams was determined, as shown in the table 1.

Table 1 Thermal conductivity of polyurethane foam of different DEG/ETA mass ratio

\begin{tabular}{cc}
\hline formulations & Thermal Conductivity $\mathrm{W} / \mathrm{m} \cdot \mathrm{K}$ \\
\hline DEG/ETA(3/1) & 0.01483 \\
DEG/ETA $(2 / 1)$ & 0.01425 \\
DEG/ETA $(1 / 1)$ & 0.01358 \\
DEG/ETA $(1 / 2)$ & 0.01542 \\
DEG/ETA(1/3) & 0.01268 \\
DEG/ETA $(1 / 3)($ adding GF $1.5 \%)$ & 0.01143 \\
\hline
\end{tabular}

It is known that the thermal conductivity standard of polyurethane foam is lower than $0.03 \mathrm{~W} / \mathrm{m} \cdot \mathrm{K}$, and the thermal conductivities of the prepared foam all meet the requirements of the standard, among which the foams (DEG/ETA $=1 / 3$, mass radio) especially the one adding glass fiber have the lowest thermal conductivity, and great heat preservation. 


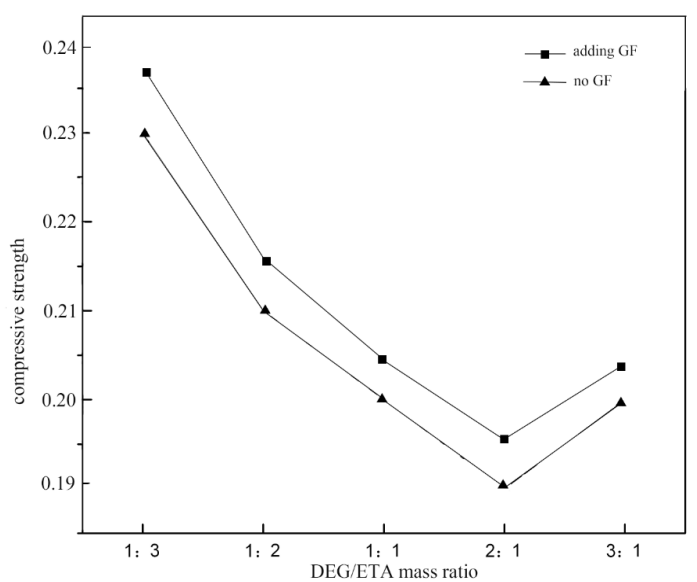

Fig. 4 Compressive strength of the foams

The prepared foam was cut into specimens in $50 \mathrm{~mm} \times 50 \mathrm{~mm} \times 50 \mathrm{~mm}$ according to the test standard, and the compressive strength was measured by the instrument to distinguish the strength of different foams.

According to the Fig. 4, with the increase of ETA content, the compressive strength of the foam gradually decrease. When the mass ratio of DEG/ETA is $1 / 3$, the foam strength is $0.27 \mathrm{MPa}$, at this time, the cells are regular hexagon and the skeleton is thick, and the crosslink density is large, so it has good compressive strength. The prepared foam can fully meet the national standards and has good compression resistance.

\section{Conclusion}

The two-component glass fiber modified waste PU/glass fiber composite materials were successfully prepared, and the results of a series of tests showed that:

1. Composite materials have low density, high compressive resistance and good thermal conductivity.

2. It was found that for the decomposition product(DEG/ETA=1/3, mass ratio), the flowability is the best, and the molecules are uniform. The polyurethane rigid foam with great pressure resistance was obtained.

3. When $1.5 \%$ glass fiber was added, a foam sample with a density of $44 \mathrm{~kg} / \mathrm{m}^{3}$, a compression strength of $0.27 \mathrm{Mpa}$ and a thermal conductivity of $0.01143 \mathrm{~W} / \mathrm{m} \cdot \mathrm{K}$ can be obstained, which has great performance.

\section{Acknowledgements}

The authors gratefully acknowledge support from Heilongjiang Provincial Department of Education Project (135109214) and Qiqihar Science and Technology Bureau Project (GYGG-201504).

\section{References}

[1] Mir Mohammad Alavi Nikje, Mahin Nikrah. Chemical Recycling and Liquefaction of Rigid Polyurethane Foam Wastes through Microwave Assisted Glycolysis Process[J]. Journal of Macromolecular Science Part A, 2007, 44(6):613-617.

[2] Murai M,Takagi T. Recycling of polyurethane foam refrigerator insulation[J]. Mitsubishi. Electr. Adv.2003,10:104-106. 
[3] Nikje A,Haghshenas M,Garmarudi A B.Preparation and application of glycolysed polyurethane integral skin foams recyclate from automotive wastes[J]. Polym. Bull.2006,56:257-265. ocess[J]. J.Macromol.Sci.A.2007,44:613-617.

[4] Wu C H, Chang C Y, Cheng C M, Huang H C. Glycolysis of waste flexible polyurethane foam [J]. Polym. Degrad. Stabil. 2003, 80:103-111.

[5] Hynek B,Jiri R,Petr Het al.Glycolysis of flexible polyurethanefoam in recycling of car seats. Polymers for Advanced Technologies . 2007.

[6] WeissKD. Paint and coatings:A mature industry in transition.Prog Polym Sci.1997; 22:203-245. 